\title{
Riflessioni sulla scrittura migrante
}

\section{Barbara Serdakowski}

\section{(2) OpenEdition}

\section{Journals}

Edizione digitale

URL: https://journals.openedition.org/cher/11138

DOI: 10.4000/cher.11138

ISSN: 2803-5992

\section{Editore}

Presses universitaires de Strasbourg

\section{Edizione cartacea}

Data di pubblicazione: 30 juin 2013

Paginazione: 89-92

ISBN: 978-2-35410-054-4

ISSN: 1968-035X

Notizia bibliografica digitale

Barbara Serdakowski, «Riflessioni sulla scrittura migrante», reCHERches [Online], 10 | 2013, online dal 08 février 2022, consultato il 10 février 2022. URL: http://journals.openedition.org/cher/11138 ; DOI: https://doi.org/10.4000/cher.11138

\section{(ब๑)}

Ce(tte) œuvre est mise à disposition selon les termes de la Licence Creative Commons Attribution Pas d'Utilisation Commerciale - Partage dans les Mêmes Conditions 4.0 International. 


\title{
Riflessioni sulla scrittura migrante
}

\author{
BARBARA SERDAKOWSKI \\ Scrittrice
}

La traduzione non è un'eco, è una lingua che lega le altre mille, si insinua tra le parole, mobile e cangiante, adattabile, penetra nel tessuto, si intreccia, si sedimenta, appartiene.

Der anni ho scritto poesie in più lingue perché ognuna rappresentava una parte di me, non riuscivo a esprimermi pienamente se non con una pluralità di lingue.

Si parla di scrittura migrante, di scrittori migranti e questo termine sembra urtare molti. Per quel che mi riguarda credo che ci sia stato un utilizzo errato di questa parola, giacché se usiamo il termine inglese migrant writer, i connotati sono ben diversi. Credo fermamente che gli studiosi, i letterati, gli scrittori abbiano una grande responsabilità nell'utilizzo di una parola nuova, soprattutto se deve poi determinare in qualche modo una categoria di persone, a maggior ragione se poi sono scrittori. Abbiamo la responsabilità di ciò che facciamo delle parole, di come vengono trasmesse nel tempo. Più che cambiare le parole, come suggeriscono in molti, io direi che sarebbe necessario ristabilirne il significato.

La scrittura migrante è un fenomeno che viene definito in contrapposizione non alla scrittura come tale, ma alla letteratura prodotta da scrittori che hanno viaggiato. Non si tratta quindi di una "subcategoria" di scrittori, né di una differenziazione tra scrittore tout court e scrittore migrante.

Ci sono stati un entusiasmo e una curiosità un po' morbosa per quello che la scrittura migrante poteva dare. Un certo tipo di voyeurismo per la narrativa prodotta come testimonianza di percorsi di vita sofferenti, di 
tragedie, di orrori di ogni genere. Ma anche qui vanno fatte distinzioni, come si fa con la letteratura prodotta da scrittori stanziali. C’è la diaristica, la narrativa di testimonianza e non sempre questi scritti vengono prodotti da scrittori veri e propri. Spesso infatti sono racconti, più o meno ben scritti, opera di persone che hanno avuto una necessità temporanea di esprimere il loro percorso di vita, la loro sofferenza, o altro, attraverso la scrittura, ma non per questo sono diventati poi scrittori. Credo che queste distinzioni siano ormai abbastanza chiare e gli stessi protagonisti della letteratura di testimonianza spesso non si definiscono scrittori.

La differenza qui è tra scrittore migrante, cioè uno scrittore che ha avuto un percorso di vita che include lo spostamento in più posti geografici, con questo alterando la sua percezione della vita, arricchendo il suo vocabolario, le sue immagini, metafore, alterando per sempre il suo percorso nella scrittura e scrittore stanziale cioè quello scrittore che per scelta o circostanze di vita non ha avuto percorsi che l'hanno portato a viaggiare, emigrare o abitare in altri posti del mondo. E quindi secondo me molte delle polemiche si basano su un errore interpretativo e l'offesa di sentirsi discriminati, e non trattati a pieno titolo come scrittori, è forse ingiustamente percepita. Ovviamente questa distinzione ci arriva anche dall'altra parte. Lo scrittore stanziale, abituato per diritto ad appartenere alla categoria degli scrittori senza aggettivo, percepisce lo scrittore migrante come appartenente a una nicchia, senza rendersi conto che la distinzione non è linguistica, ma di percorso di vita.

Lo scrittore stanziale non ha né la scelta, né la colpa, dell'appartenenza alla sua stessa letteratura. Non ha quindi domande da farsi. Scrive e basta.

Lo scrittore che viene da altri posti si trova quasi sempre davanti ad una scelta, condizionata dalla sua realtà, dalla sua convinzione, dalla sua indole. Ci sono scrittori che hanno l'indole migrante e altri no. Ci sono infatti scrittori migranti che dell'esperienza di migrazione avrebbero volentieri fatto a meno, come ci sono scrittori stanziali che non desidererebbero altro che avere l'opportunità di viaggiare e di scoprire altre realtà nel mondo.

Per quanto ho potuto osservare, le due scelte principali da fare in terra altrui sono: integrarsi nella scrittura contemporanea italiana e lottare contro l'emarginazione e la diffidenza reclamando a gran voce il proprio posto tra gli scrittori del luogo adottato; appartenere al gruppo mondiale e, soprattutto in questo caso europeo, di scrittori migranti del quale fanno parte tutti i creatori che hanno percorsi di vita non stanziale, lottando in questo caso contro il pericolo dello sfruttamento merceologico che tende 
a voler mettere troppo l'accento sulle tematiche della migrazione, sui ponti tra culture e di allontanarsi per motivi di interessi vari dall'obbiettivo e dai meriti letterari.

Per quel che mi riguarda, il termine scrittore migrante, scrittura migrante è valido, ma soltanto nel suo significato antagonista a scrittore stanziale, scrittura stanziale, e quindi se prodotto in italiano appartenente per automatismo e per definizione alla scrittura nazionale del paese.

In Canada ero considerata un'allofona, e quando scrivevo appartenevo alla scrittura francofona, come opposizione alla scrittura anglofona o alla scrittura allofona tradotta dal francese o dall'inglese. Le due lingue erano considerate valide dato che ufficialmente il Canada è bilingue e per di più il multiculturalismo è considerato una forma di struttura sociale, di tessuto nazionale. Ognuno può essere quello che vuole, ma poi per la divulgazione deve scegliere una delle due lingue ufficiali o l'originale con la sua traduzione verso una di queste due lingue. Faccio questo confronto perché in qualsiasi lingua venisse prodotta, la mia scrittura era considerata scrittura "canadese".

Credo che per tutti i fenomeni analizzati in un determinato periodo storico per qualsiasi motivo, sia giusto intervenire, confrontarsi, definire correttamente le cose, correggere gli eccessi e farlo in modo serio, attento e responsabile e a questo proposito vorrei concludere con un componimento che riguarda la lingua, la mia lingua, dal titolo La morta:

È inutile parlare quando tutto tace

Quando il suono cessa come la vita, all'improvviso

Ti chini per toccarmi col petto teso

Hai mani bagnate e profumo di cipolla

Ci sono, nella casa della morta, odori di cucina.

Tu scèles mes lèvres de salive

Sigilli le mie labbra con saliva

De la salive vieille que tu tenais serrée entre les dents

Della vecchia saliva che tenevi stretta tra i denti

Irresistente come la carta il tuo sguardo parte

Sono una morta lontana ormai, nei tuoi occhi spenti.

Brush my eyelashes with both your cheeks, Strofinami le ciglia con entrambe le guance 
Once more without good bye Una volta ancora senza arrivederci

Già ti vesti di neve e porti fiori finti fiori da non dover tornare spesso Sono ossa sottili tra le tue mani Corri lontano dalle onde dei miei pensieri, inciampi, sono fili.

¿Che harás con nuestras oras perdidas?

Cosa farai con le nostre ore perse?

Quelle ore di niente

Solo tue, solo mie

Nella casa della morta non ci sono più io Ci sei tu adesso con gli altri, E mangiate in fretta sul mio destino Nelle mie scarpe strette bevete in allegria triste

Tears of wine and onion soup. Lacrime di vino e zuppa di cipolle. 